\title{
WEAK GALERKIN FINITE ELEMENT METHODS FOR THE BIHARMONIC EQUATION ON POLYTOPAL MESHES
}

\author{
LIN MU*, JUNPING WANG ${ }^{\dagger}$, AND XIU YE
}

\begin{abstract}
A new weak Galerkin (WG) finite element method is introduced and analyzed in this paper for the biharmonic equation in its primary form. This method is highly robust and flexible in the element construction by using discontinuous piecewise polynomials on general finite element partitions consisting of polygons or polyhedra of arbitrary shape. The resulting WG finite element formulation is symmetric, positive definite, and parameter-free. Optimal order error estimates in a discrete $H^{2}$ norm is established for the corresponding WG finite element solutions. Error estimates in the usual $L^{2}$ norm are also derived, yielding a sub-optimal order of convergence for the lowest order element and an optimal order of convergence for all high order of elements. Numerical results are presented to confirm the theory of convergence under suitable regularity assumptions.
\end{abstract}

Key words. weak Galerkin, finite element methods, weak Laplacian, biharmonic equations, polyhedral meshes.

AMS subject classifications. Primary, 65N15, 65N30, 74K20; Secondary, 35B45, 35J50, $35 \mathrm{~J} 35$

1. Introduction. This paper is concerned with numerical methods for the biharmonic equation with boundary conditions. For simplicity, consider the model problem that seeks an unknown function $u=u(x)$ satisfying

$$
\begin{array}{rlrl}
\Delta^{2} u & =f, & & \text { in } \Omega, \\
u & =\zeta, & & \text { on } \partial \Omega, \\
\frac{\partial u}{\partial n}=\phi & & \text { on } \partial \Omega,
\end{array}
$$

where $\Delta$ is the Laplacian operator, $\zeta=\zeta(x)$ and $\phi=\phi(x)$ are given functions defined on the boundary of the domain $\Omega$. Assume that the domain $\Omega$ is open bounded with a Lipschitz continuous boundary $\partial \Omega$ in $\mathbb{R}^{d}, d=2,3$.

A natural variational formulation for the biharmonic equation (1.1) with Dirichlet and Neumann boundary conditions (1.2) and (1.3) seeks $u \in H^{2}(\Omega)$ satisfying $\left.u\right|_{\partial \Omega}=$ $\zeta$ and $\left.\frac{\partial u}{\partial n}\right|_{\partial \Omega}=\phi$ such that

$$
(\Delta u, \Delta v)=(f, v), \quad \forall v \in H_{0}^{2}(\Omega),
$$

where $H_{0}^{2}(\Omega)$ is a subspace of the Sobolev space $H^{2}(\Omega)$ consisting of functions with vanishing value and normal derivative on $\partial \Omega$. Based on the variational form (1.4), one

\footnotetext{
*Department of Mathematics, University of Arkansas at Little Rock, Little Rock, AR 72204

$\dagger^{\dagger}$ Division of Mathematical Sciences, National Science Foundation, Arlington, VA 22230 (jwang@ nsf.gov). The research of Wang was supported by the NSF IR/D program, while working at National Science Foundation. However, any opinion, finding, and conclusions or recommendations expressed in this material are those of the author and do not necessarily reflect the views of the National Science Foundation,

${ }^{\ddagger}$ Department of Mathematics, University of Arkansas at Little Rock, Little Rock, AR 72204 (xxye@ualr.edu). This research was supported in part by National Science Foundation Grant DMS1115097.
} 
may design various conforming finite element schemes for (1.1)-(1.3) by constructing finite element spaces as subspaces of $H^{2}(\Omega)$. It is known that $H^{2}$-conforming methods essentially require $C^{1}$-continuous piecewise polynomials on a prescribed finite element partition, which imposes an enormous difficulty in practical computation. Due to the complexity in the construction of $C^{1}$-continuous elements, $H^{2}$-conforming finite element methods are rarely used in practice for solving the biharmonic equation.

As an alternative approach, nonconforming and discontinuous Galerkin finite element methods have been developed for solving the biharmonic equation over the last several decades. The Morley element [6] is a well-known example of nonconforming element for the biharmonic equation by using piecewise quadratic polynomials. Recently, a $C^{0}$ interior penalty method was studied in $[2,3]$. In [8], a $h p$-version interior-penalty discontinuous Galerkin method was developed for the biharmonic equation. To avoid the use of $C^{1}$-elements, mixed methods have been developed for the biharmonic equation by reducing the fourth order problem to a system of two second order equations $[1,4,5,7,10]$.

In this paper, we will develop a highly flexible and robust weak Galerkin finite element method for the biharmonic equation that allows the use of generalized approximating functions on general partitions consisting of polygons or polyhedra of arbitrary shape with certain shape regularity. The weak Galerkin method refers to a numerical technique for partial differential equations where differential operators are interpreted and approximated as distributions over a set of generalized functions. The method/idea was first introduced in [11] for second order elliptic equations, and the concept was further developed in $[12,13,9]$. By design, weak Galerkin methods use generalized and/or discontinuous approximating functions on general meshes to overcome the barrier in the construction of "smooth" finite element functions.

Intuitively, a weak Galerkin finite element scheme for the biharmonic equation (1.1)-(1.3) can be derived by replacing the differential operator $\Delta$ in (1.4) by a discrete weak Laplacian, denoted by $\Delta_{w}$. However, such a straight forward replacement may not work without including a mechanism that enforces a certain weak continuity of the underlying approximating functions. A weak continuity can be realized by introducing an appropriately defined stabilizer, denoted as $s(\cdot, \cdot)$. Formally, our weak Galerkin finite element method for (1.1)-(1.3) can be described by seeking a finite element function $u_{h}$ satisfying

$$
\left(\Delta_{w} u_{h}, \Delta_{w} v\right)+s\left(u_{h}, v\right)=(f, v)
$$

for all testing functions $v$. The goal of the paper is to specify all the details for (1.5), and further justify the rigorousness of the method by establishing a mathematical convergence theory.

The paper is organized as follows. In Section 2, we introduce some standard notations for Sobolev spaces. A weak Laplacian operator and its discrete version will be introduced in Section 3. In Section 4, we shall present two WG finite element schemes for the biharmonic equation (1.1)-(1.3). In Section 5, we shall introduce some local $L^{2}$ projection operators and then derive some approximation properties which are useful in a convergence analysis. In Section 6, we shall establish optimal order error estimates for the WG finite element approximation in a $H^{2}$-equivalent discrete norm. In Section 7, we shall derive an error estimate for the WG-FEM approximation in the usual $L^{2}$ norm. Results from two numerical experiments are 
reported in Section 8. Finally, we provide some technical results in the appendix that are critical in dealing with finite element functions on arbitrary polygons/polyhedra.

2. Preliminaries and Notations. Let $D$ be any open bounded domain with Lipschitz continuous boundary in $\mathbb{R}^{d}, d=2,3$. We use the standard definition for the Sobolev space $H^{s}(D)$ and the associated inner product $(\cdot, \cdot)_{s, D}$, norm $\|\cdot\|_{s, D}$, and seminorm $|\cdot|_{s, D}$ for any $s \geq 0$. For example, for any integer $s \geq 0$, the seminorm $|\cdot|_{s, D}$ is given by

$$
|v|_{s, D}=\left(\sum_{|\alpha|=s} \int_{D}\left|\partial^{\alpha} v\right|^{2} d D\right)^{\frac{1}{2}}
$$

with the usual notation

$$
\alpha=\left(\alpha_{1}, \ldots, \alpha_{d}\right), \quad|\alpha|=\alpha_{1}+\ldots+\alpha_{d}, \quad \partial^{\alpha}=\prod_{j=1}^{d} \partial_{x_{j}}^{\alpha_{j}} .
$$

The Sobolev norm $\|\cdot\|_{m, D}$ is given by

$$
\|v\|_{m, D}=\left(\sum_{j=0}^{m}|v|_{j, D}^{2}\right)^{\frac{1}{2}} .
$$

The space $H^{0}(D)$ coincides with $L^{2}(D)$, for which the norm and the inner product are denoted by $\|\cdot\|_{D}$ and $(\cdot, \cdot)_{D}$, respectively. When $D=\Omega$, we shall drop the subscript $D$ in the norm and inner product notation.

The space $H(\operatorname{div} ; D)$ is defined as the set of vector-valued functions on $D$ which, together with their divergence, are square integrable; i.e.,

$$
H(\operatorname{div} ; D)=\left\{\mathbf{v}: \mathbf{v} \in\left[L^{2}(D)\right]^{d}, \nabla \cdot \mathbf{v} \in L^{2}(D)\right\} .
$$

The norm in $H($ div; $D)$ is defined by

$$
\|\mathbf{v}\|_{H(\operatorname{div} ; D)}=\left(\|\mathbf{v}\|_{D}^{2}+\|\nabla \cdot \mathbf{v}\|_{D}^{2}\right)^{\frac{1}{2}} .
$$

3. Weak Laplacian and Discrete Weak Laplacian. For the biharmonic problem (1.1)-(1.3) with the variational form (1.4), the principle differential operator is the Laplacian $\Delta$. Thus, we shall introduce a weak Laplacian operator defined on a class of discontinuous functions. For numerical purposes, we will define a discrete version of the weak Laplacian in polynomial subspaces.

Let $T$ be any polygonal or polyhedral domain with boundary $\partial T$. By a weak function on the region $T$ we mean a function $v=\left\{v_{0}, v_{b}, \mathbf{v}_{g}\right\}$ such that $v_{0} \in L^{2}(T)$, $v_{b} \in H^{\frac{1}{2}}(\partial T)$, and $\mathbf{v}_{g} \cdot \mathbf{n} \in H^{-\frac{1}{2}}(\partial T)$, where $\mathbf{n}$ is the outward normal direction of $T$ on its boundary. The first and second components $v_{0}$ and $v_{b}$ can be understood as the value of $v$ in the interior and on the boundary of $T$. The third component $\mathbf{v}_{g}$ intends to represent the gradient of $v$ on the boundary of $T$. Note that $v_{b}$ and $\mathbf{v}_{g}$ may not be necessarily related to the trace of $v_{0}$ and $\nabla v_{0}$ on $\partial T$, respectively. 
Denote by $W(T)$ the space of all weak functions on $T$; i.e.,

$$
W(T)=\left\{v=\left\{v_{0}, v_{b}, \mathbf{v}_{g}\right\}: v_{0} \in L^{2}(T), v_{b} \in H^{\frac{1}{2}}(\partial T), \mathbf{v}_{g} \cdot \mathbf{n} \in H^{-\frac{1}{2}}(\partial T)\right\} .
$$

Let $\langle\cdot, \cdot\rangle_{\partial T}$ be the inner product in $L^{2}(\partial T)$. Define $G_{2}(T)$ by

$$
G_{2}(T)=\left\{\varphi: \varphi \in H^{1}(T), \Delta \varphi \in L^{2}(T)\right\} .
$$

For any $\varphi \in G_{2}(T)$, we have $\nabla \varphi \in H(d i v, T)$, and hence $\nabla \varphi \cdot \mathbf{n} \in H^{-\frac{1}{2}}(\partial T)$.

Definition 3.1. The dual of $L^{2}(T)$ can be identified with itself by using the standard $L^{2}$ inner product as the action of linear functionals. With a similar interpretation, for any $v \in W(T)$, the weak Laplacian of $v=\left\{v_{0}, v_{b}, \mathbf{v}_{g}\right\}$ is defined as a linear functional $\Delta_{w} v$ in the dual space of $G_{2}(T)$ whose action on each $\varphi \in G_{2}(T)$ is given by

$$
\left(\Delta_{w} v, \varphi\right)_{T}=\left(v_{0}, \Delta \varphi\right)_{T}-\left\langle v_{b}, \nabla \varphi \cdot \mathbf{n}\right\rangle_{\partial T}+\left\langle\mathbf{v}_{g} \cdot \mathbf{n}, \varphi\right\rangle_{\partial T},
$$

where $\mathbf{n}$ is the outward normal direction to $\partial T$.

The Sobolev space $H^{2}(T)$ can be embedded into the space $W(T)$ by an inclusion map $i_{W}: H^{2}(T) \rightarrow W(T)$ defined as follows

$$
i_{W}(\phi)=\left\{\left.\phi\right|_{T},\left.\phi\right|_{\partial T},\left.\nabla \phi\right|_{\partial T}\right\}, \quad \phi \in H^{2}(T) .
$$

With the help of the inclusion map $i_{W}$, the Sobolev space $H^{2}(T)$ can be viewed as a subspace of $W(T)$ by identifying each $\phi \in H^{2}(T)$ with $i_{W}(\phi)$. Analogously, a weak function $v=\left\{v_{0}, v_{b}, \mathbf{v}_{g}\right\} \in W(T)$ is said to be in $H^{2}(T)$ if it can be identified with a function $\phi \in H^{2}(T)$ through the above inclusion map. It is not hard to see that the weak Laplacian is identical with the strong Laplacian in $H^{2}(T)$; i.e., $\Delta_{w} v=\Delta v$ for all functions $v \in H^{2}(T)$.

Next, we introduce a discrete weak Laplacian operator by approximating $\Delta_{w}$ in a polynomial subspace of the dual of $G_{2}(T)$. To this end, for any non-negative integer $r \geq 0$, denote by $P_{r}(T)$ the set of polynomials on $T$ with degree no more than $r$. A discrete weak Laplacian operator, denoted by $\Delta_{w, r, T}$, is defined as the unique polynomial $\Delta_{w, r, T} v \in P_{r}(T)$ satisfying the following equation

$$
\left(\Delta_{w, r, T} v, \varphi\right)_{T}=\left(v_{0}, \Delta \varphi\right)_{T}-\left\langle v_{b}, \nabla \varphi \cdot \mathbf{n}\right\rangle_{\partial T}+\left\langle\mathbf{v}_{g} \cdot \mathbf{n}, \varphi\right\rangle_{\partial T}, \quad \forall \varphi \in P_{r}(T) .
$$

4. Weak Galerkin Finite Element Schemes. Let $\mathcal{T}_{h}$ be a partition of the domain $\Omega$ into polygons in $2 \mathrm{D}$ or polyhedra in $3 \mathrm{D}$. Assume that $\mathcal{T}_{h}$ is shape regular in the sense as defined in [12] (seel also Appendix A). Denote by $\mathcal{E}_{h}$ the set of all edges or flat faces in $\mathcal{T}_{h}$, and let $\mathcal{E}_{h}^{0}=\mathcal{E}_{h} \backslash \partial \Omega$ be the set of all interior edges or flat faces.

For any given integer $k \geq 2$, denote by $W_{k}(T)$ the discrete weak function space given by

$$
W_{k}(T)=\left\{\left\{v_{0}, v_{b}, \mathbf{v}_{g}\right\}: v_{0} \in P_{k}(T), v_{b} \in P_{k}(e), \mathbf{v}_{g} \in\left[P_{k-1}(e)\right]^{d}, e \subset \partial T\right\} .
$$

By patching $W_{k}(T)$ over all the elements $T \in \mathcal{T}_{h}$ through a common value on the interface $\mathcal{E}_{h}^{0}$, we arrive at a weak finite element space $V_{h}$ given by

$$
V_{h}=\left\{\left\{v_{0}, v_{b}, \mathbf{v}_{g}\right\}:\left.\left\{v_{0}, v_{b}, \mathbf{v}_{g}\right\}\right|_{T} \in W_{k}(T), \forall T \in \mathcal{T}_{h}\right\} .
$$


Denote by $\Lambda_{h}$ the trace of $V_{h}$ on $\partial \Omega$ from the component $v_{b}$. It is clear that $\Lambda_{h}$ consists of piecewise polynomials of degree $k$. Similarly, denote by $\Upsilon_{h}$ the trace of $V_{h}$ from the normal component of $\mathbf{v}_{g}$ as piecewise polynomials of degree $k-1$. Denote by $V_{h}^{0}$ the subspace of $V_{h}$ with vanishing traces; i.e.,

$$
V_{h}^{0}=\left\{v=\left\{v_{0}, v_{b}, \mathbf{v}_{g}\right\} \in V_{h},\left.v_{b}\right|_{e}=0,\left.\mathbf{v}_{g} \cdot \mathbf{n}\right|_{e}=0, e \subset \partial T \cap \partial \Omega\right\} .
$$

Denote by $\Delta_{w, k-2}$ the discrete weak Laplacian operator on the finite element space $V_{h}$ computed by using (3.3) on each element $T$ for $k \geq 2$; i.e.,

$$
\left.\left(\Delta_{w, k-2} v\right)\right|_{T}=\Delta_{w, k-2, T}\left(\left.v\right|_{T}\right), \quad \forall v \in V_{h} .
$$

For simplicity, we shall drop the subscript $k-2$ in the notation $\Delta_{w, k-2}$ for the discrete weak Laplacian. We also introduce the following notation

$$
\left(\Delta_{w} v, \Delta_{w} w\right)_{h}:=\sum_{T \in \mathcal{T}_{h}}\left(\Delta_{w} v, \Delta_{w} w\right)_{T} .
$$

4.1. Algorithm I. For any $u_{h}=\left\{u_{0}, u_{b}, \mathbf{u}_{g}\right\}$ and $v=\left\{v_{0}, v_{b}, \mathbf{v}_{g}\right\}$ in $V_{h}$, we introduce a bilinear form as follows

$$
s\left(u_{h}, v\right):=\sum_{T \in \mathcal{T}_{h}} h_{T}^{-1}\left\langle\nabla u_{0}-\mathbf{u}_{g}, \nabla v_{0}-\mathbf{v}_{g}\right\rangle_{\partial T}+\sum_{T \in \mathcal{T}_{h}} h_{T}^{-3}\left\langle u_{0}-u_{b}, v_{0}-v_{b}\right\rangle_{\partial T} .
$$

Weak Galerkin Algorithm 1. A numerical approximation for (1.1)-(1.3) can be obtained by seeking $u_{h}=\left\{u_{0}, u_{b}, \mathbf{u}_{g}\right\} \in V_{h}$ satisfying $u_{b}=Q_{b} \zeta$ and $\mathbf{u}_{g} \cdot \mathbf{n}=Q_{g n} \phi$ on $\partial \Omega$ and the following equation:

$$
\left(\Delta_{w} u_{h}, \Delta_{w} v\right)_{h}+s\left(u_{h}, v\right)=\left(f, v_{0}\right), \quad \forall v=\left\{v_{0}, v_{b}, \mathbf{v}_{g}\right\} \in V_{h}^{0},
$$

where $Q_{b} \zeta$ is the standard $L^{2}$ projection onto the trace space $\Lambda_{h}$ and $Q_{g n} \phi$ is the $L^{2}$ projection onto the normal component of the gradient trace space $\Upsilon_{h}$.

The following is a useful observation concerning the finite element space $V_{h}^{0}$.

Lemma 4.1. For any $v \in V_{h}^{0}$, let $\|v\|$ be given as follows

$$
\|v\|^{2}=\left(\Delta_{w} v, \Delta_{w} v\right)_{h}+s(v, v) .
$$

Then, $\|\cdot\|||$ defines a norm in the linear space $V_{h}^{0}$.

Proof. We shall only verify the positivity property for $\|\cdot\| \cdot \|$. To this end, assume that $\|v\|=0$ for some $\left\{v_{0}, v_{b}, \mathbf{v}_{g}\right\} \in V_{h}^{0}$. It follows that $\Delta_{w} v=0, v_{0}=v_{b}$, and $\nabla v_{0}=\mathbf{v}_{g}$ on each element $T$ or $\partial T$, as appropriate. We claim that $\Delta v_{0}=0$ holds true locally on each element $T$. To this end, for any $\varphi \in P_{k-2}(T)$ we use $\Delta_{w} v=0$ and the definition (3.3) to obtain

$$
\begin{aligned}
0 & =\left(\Delta_{w} v, \varphi\right)_{T} \\
& =\left(v_{0}, \Delta \varphi\right)_{T}-\left\langle v_{b}, \nabla \varphi \cdot \mathbf{n}\right\rangle_{\partial T}+\left\langle\mathbf{v}_{g} \cdot \mathbf{n}, \varphi\right\rangle_{\partial T} \\
& =\left(\Delta v_{0}, \varphi\right)_{T}+\left\langle v_{0}-v_{b}, \nabla \varphi \cdot \mathbf{n}\right\rangle_{\partial T}+\left\langle\mathbf{v}_{g} \cdot \mathbf{n}-\nabla v_{0} \cdot \mathbf{n}, \varphi\right\rangle_{\partial T} \\
& =\left(\Delta v_{0}, \varphi\right)_{T},
\end{aligned}
$$


where we have used the fact that $v_{0}-v_{b}=0$ and $\nabla v_{0}-\mathbf{v}_{g}=0$ in the last equality. The identity (4.5) implies that $\Delta v_{0}=0$ holds true locally on each element $T$. This, together with $v_{0}=v_{b}$ and $\nabla v_{0}=\mathbf{v}_{g}$ on $\partial T$, shows that $v$ is a smooth harmonic function globally on $\Omega$. The boundary condition of $v_{b}=0$ then implies that $v \equiv 0$ on $\Omega$, which completes the proof.

LEMma 4.2. The weak Galerkin finite element scheme (4.2) has a unique solution.

Proof. Let $\mathbf{u}_{h}^{(1)}$ and $\mathbf{u}_{h}^{(2)}$ be two solutions of the weak Galerkin finite element scheme (4.2). It is clear that the difference $\mathbf{e}_{h}=\mathbf{u}_{h}^{(1)}-\mathbf{u}_{h}^{(2)}$ is a finite element function in $V_{h}^{0}$ satisfying

$$
\left(\Delta_{w} \mathbf{e}_{h}, \Delta_{w} v\right)_{h}+s\left(\mathbf{e}_{h}, v\right)=0, \quad \forall v=\left\{v_{0}, v_{b}, \mathbf{v}_{g}\right\} \in V_{h}^{0} .
$$

By setting $v=\mathbf{e}_{h}$ in (4.6) we obtain

$$
\left(\Delta_{w} \mathbf{e}_{h}, \Delta_{w} \mathbf{e}_{h}\right)_{h}+s\left(\mathbf{e}_{h}, \mathbf{e}_{h}\right)=0 .
$$

It follows from Lemma 4.1 that $\mathbf{e}_{h} \equiv 0$. This shows that $\mathbf{u}_{h}^{(1)}=\mathbf{u}_{h}^{(2)}$.

4.2. Algorithm II. Here we describe another weak Galerkin finite element scheme that has less number of unknowns than (4.2). This second WG-FEM scheme is formulated in a subspace of $V_{h}$, denoted by $\tilde{V}_{h}$, that uses only the normal component of the vector $\mathbf{v}_{g}$ for any $v=\left\{v_{0}, v_{b}, \mathbf{v}_{g}\right\} \in V_{h}$. To be more precise, let us introduce a set of normal directions on $\mathcal{E}_{h}$ as follows

$$
\mathcal{D}_{h}=\left\{\mathbf{n}_{e}: \mathbf{n}_{e} \text { is unit and normal to } e, e \in \mathcal{E}_{h}\right\} .
$$

The weak Galerkin finite element space $\tilde{V}_{h}$ is given as follows

$$
\tilde{V}_{h}=\left\{v=\left\{v_{0}, v_{b}, v_{g} \mathbf{n}_{e}\right\}: v_{0} \in P_{k}(T), v_{b} \in P_{k}(e), v_{g} \in P_{k-1}(e), e \subset \partial T\right\},
$$

where $v_{g}$ can be viewed as an approximation of $\nabla v \cdot \mathbf{n}_{e}$. Denote by $\tilde{V}_{h}^{0}$ the subspace of $V_{h}$ with vanishing traces; i.e.,

$$
\tilde{V}_{h}^{0}=\left\{v=\left\{v_{0}, v_{b}, v_{g} \mathbf{n}_{e}\right\} \in V_{h},\left.v_{b}\right|_{e}=0,\left.v_{g}\right|_{e}=0, e \subset \partial T \cap \partial \Omega\right\} .
$$

For any $u_{h}=\left\{u_{0}, u_{b}, u_{g} \mathbf{n}_{e}\right\}$ and $v=\left\{v_{0}, v_{b}, v_{g} \mathbf{n}_{e}\right\}$ in $\tilde{V}_{h}$, we introduce a bilinear form as follows

$\tilde{s}\left(u_{h}, v\right):=\sum_{T \in \mathcal{T}_{h}} h_{T}^{-1}\left\langle\nabla u_{0} \cdot \mathbf{n}_{e}-u_{g}, \nabla v_{0} \cdot \mathbf{n}_{e}-v_{g}\right\rangle_{\partial T}+\sum_{T \in \mathcal{T}_{h}} h_{T}^{-3}\left\langle u_{0}-u_{b}, v_{0}-v_{b}\right\rangle_{\partial T}$.

Weak Galerkin Algorithm 2. A numerical approximation for (1.1)-(1.3) can be obtained by seeking $u_{h}=\left\{u_{0}, u_{b}, u_{g} \mathbf{n}_{e}\right\} \in \tilde{V}_{h}$ satisfying $u_{b}=Q_{b} \zeta$ and $u_{g}=Q_{g n} \phi$ on $\partial \Omega$ and the following equation:

$$
\left(\Delta_{w} u_{h}, \Delta_{w} v\right)_{h}+\tilde{s}\left(u_{h}, v\right)=\left(f, v_{0}\right), \quad \forall v=\left\{v_{0}, v_{b}, v_{g} \mathbf{n}_{e}\right\} \in \tilde{V}_{h}^{0},
$$

where $Q_{b} \zeta$ is the standard $L^{2}$ projection onto the trace space $\Lambda_{h}$ and $Q_{g n} \phi$ is the $L^{2}$ projection onto the normal component of the gradient trace space $\Upsilon_{h}$.

Like (4.2), the WG-FEM scheme (4.9) can be proved to have one and only one solution in the corresponding finite element space. Details are left to interested readers for a verification. 
5. $L^{2}$ Projections and Approximation Properties. For each element $T$, denote by $Q_{0}$ the $L^{2}$ projection onto $P_{k}(T), k \geq 2$. For each edge/face $e \subset \partial T$, denote by $Q_{b}$ and $\mathbf{Q}_{g}$ the $L^{2}$ projection onto $P_{k}(e)$ and $\left[P_{k-1}(e)\right]^{d}$, respectively. Now for any $u \in H^{2}(\Omega)$, we can define a projection into the finite element space $V_{h}$ such that on the element $T$

$$
Q_{h} u=\left\{Q_{0} u, Q_{b} u, \mathbf{Q}_{g}(\nabla u)\right\}
$$

In addition, denote by $\mathbb{Q}_{h}$ the local $L^{2}$ projection onto $P_{k-2}(T)$.

Lemma 5.1. On each element $T \in \mathcal{T}_{h}$, the $L^{2}$ projections $Q_{h}$ and $\mathbb{Q}_{h}$ satisfy the following commutative property with the Laplacian $\Delta$ and the discrete weak Laplacian $\Delta_{w}$ :

$$
\Delta_{w}\left(Q_{h} u\right)=\mathbb{Q}_{h}(\Delta u)
$$

for all $u \in H^{2}(T)$.

Proof. For any $\tau \in P_{k-2}(T)$, it is not hard to see that

$$
\begin{aligned}
\left(\Delta_{w} Q_{h} u, \tau\right)_{T} & =\left(Q_{0} u, \Delta \tau\right)_{T}+\left\langle\mathbf{Q}_{g}(\nabla u) \cdot \mathbf{n}, \tau\right\rangle_{\partial T}-\left\langle Q_{b} u, \nabla \tau \cdot \mathbf{n}\right\rangle_{\partial T} \\
& =(u, \Delta \tau)_{T}+\langle\nabla u \cdot \mathbf{n}, \tau\rangle_{\partial T}-\langle u, \nabla \tau \cdot \mathbf{n}\rangle_{\partial T} \\
& =(\Delta u, \tau)_{T} \\
& =\left(\mathbb{Q}_{h} \Delta u, \tau\right)_{T},
\end{aligned}
$$

which implies the desired identity (5.1).

The commutative property (5.1) indicates that the discrete weak Laplacian of the $L^{2}$ projection of smooth functions is a good approximation of the Laplacian of the function itself in the classical sense. This is a nice and useful property of the discrete weak Laplacian in application to algorithm design and analysis.

The following lemma provides some approximation properties for the projection operators $Q_{h}$ and $\mathbb{Q}_{h}$.

Lemma 5.2. Let $\mathcal{T}_{h}$ be a finite element partition of $\Omega$ satisfying the shape regularity assumption as specified in [12]. Then, for any $0 \leq s \leq 2$ and $2 \leq m \leq k$ we have

$$
\begin{aligned}
& \sum_{T \in \mathcal{T}_{h}} h_{T}^{2 s}\left\|u-Q_{0} u\right\|_{s, T}^{2} \leq C h^{2(m+1)}\|u\|_{m+1}^{2}, \\
& \sum_{T \in \mathcal{T}_{h}} h_{T}^{2 s}\left\|\Delta u-\mathbb{Q}_{h} \Delta u\right\|_{s, T}^{2} \leq C h^{2(m-1)}\|u\|_{m+1}^{2} .
\end{aligned}
$$

Proof. The proof of this lemma is similar to that of Lemma 5.1 in [12], and the details are thus omitted. $\square$

Using the trace inequality (A.3) in the Appendix with $p=2$, we can derive the following estimates which are useful in the convergence analysis for the weak Galerkin finite element schemes (4.2) and (4.9). 
Lemma 5.3. Let $2 \leq m \leq k, w \in H^{\max \{m+1,4\}}(\Omega)$, and $v \in V_{h}$. There exists a constant $C$ such that the following estimates hold true:

$$
\begin{gathered}
\left(\sum_{T \in \mathcal{T}_{h}} h_{T}\left\|\Delta w-\mathbb{Q}_{h} \Delta w\right\|_{\partial T}^{2}\right)^{\frac{1}{2}} \leq C h^{m-1}\|w\|_{m+1}, \\
\left(\sum_{T \in \mathcal{T}_{h}} h_{T}^{3}\left\|\nabla\left(\Delta w-\mathbb{Q}_{h} \Delta w\right)\right\|_{\partial T}^{2}\right)^{\frac{1}{2}} \leq C h^{m-1}\left(\|w\|_{m+1}+h \delta_{m, 2}\|w\|_{4}\right), \\
\left(\sum_{T \in \mathcal{T}_{h}} h_{T}^{-1}\left\|\nabla\left(Q_{0} w\right)-\mathbf{Q}_{g}(\nabla w)\right\|_{\partial T}^{2}\right)^{\frac{1}{2}} \leq C h^{m-1}\|w\|_{m+1}, \\
\left(\sum_{T \in \mathcal{T}_{h}} h_{T}^{-3}\left\|Q_{0} w-Q_{b} w\right\|_{\partial T}^{2}\right)^{\frac{1}{2}} \leq C h^{m-1}\|w\|_{m+1} .
\end{gathered}
$$

Here $\delta_{i, j}$ is the usual Kronecker's delta with value 1 when $i=j$ and value 0 otherwise.

Proof. To derive (5.4), we use the trace inequality (A.3) and the estimate (5.3) to obtain

$$
\begin{aligned}
& \sum_{T \in \mathcal{T}_{h}} h_{T}\left\|\Delta w-\mathbb{Q}_{h} \Delta w\right\|_{\partial T}^{2} \\
\leq & C \sum_{T \in \mathcal{T}_{h}}\left(\left\|\Delta w-\mathbb{Q}_{h} \Delta w\right\|_{T}^{2}+h_{T}^{2}\left\|\nabla\left(\Delta w-\mathbb{Q}_{h} \Delta w\right)\right\|_{T}^{2}\right) \\
\leq & C h^{2 m-2}\|w\|_{m+1}^{2} .
\end{aligned}
$$

As to (5.5), we use the trace inequality (A.3) and the estimate (5.3) to obtain

$$
\begin{aligned}
& \sum_{T \in \mathcal{T}_{h}} h_{T}^{3}\left\|\nabla\left(\Delta w-\mathbb{Q}_{h} \Delta w\right)\right\|_{\partial T}^{2} \\
\leq & C \sum_{T \in \mathcal{T}_{h}}\left(h_{T}^{2}\left\|\nabla\left(\Delta w-\mathbb{Q}_{h} \Delta w\right)\right\|_{T}^{2}+h_{T}^{4}\left\|\nabla^{2}\left(\Delta w-\mathbb{Q}_{h} \Delta w\right)\right\|_{T}^{2}\right) \\
\leq & C h^{2 m-2}\left(\|w\|_{m+1}^{2}+h^{2} \delta_{m, 2}\|w\|_{4}^{2}\right) .
\end{aligned}
$$

As to (5.6), we have from the definition of $\mathbf{Q}_{g}$, the trace inequality (A.3), and the estimate (5.2) that

$$
\begin{aligned}
& \sum_{T \in \mathcal{T}_{h}} h_{T}^{-1}\left\|\nabla\left(Q_{0} w\right)-\mathbf{Q}_{g}(\nabla w)\right\|_{\partial T}^{2} \\
\leq & \sum_{T \in \mathcal{T}_{h}} h_{T}^{-1}\left\|\nabla Q_{0} w-\nabla w\right\|_{\partial T}^{2} \\
\leq & C \sum_{T \in \mathcal{T}_{h}}\left(h_{T}^{-2}\left\|\nabla Q_{0} w-\nabla w\right\|_{T}^{2}+\left\|\nabla Q_{0} w-\nabla w\right\|_{1, T}^{2}\right) \\
\leq & C h^{2 m-2}\|w\|_{m+1}^{2} .
\end{aligned}
$$


Finally, we use the definition of $Q_{b}$ and the trace inequality (A.3) to obtain

$$
\begin{aligned}
& \sum_{T \in \mathcal{T}_{h}} h_{T}^{-3}\left\|Q_{0} w-Q_{b} w\right\|_{\partial T}^{2} \leq \sum_{T \in \mathcal{T}_{h}} h_{T}^{-3}\left\|Q_{0} w-w\right\|_{\partial T}^{2} \\
\leq & C \sum_{T \in \mathcal{T}_{h}}\left(h_{T}^{-4}\left\|Q_{0} w-w\right\|_{T}^{2}+h_{T}^{-2}\left\|\nabla\left(Q_{0} w-w\right)\right\|_{T}^{2}\right) \\
\leq & C h^{2 m-2}\|w\|_{m+1}^{2} .
\end{aligned}
$$

This completes the proof of (5.7), and hence the lemma.

6. An Error Estimate in $H^{2}$. The goal here is to establish an error estimate for the WG-FEM solution $u_{h}$ arising from (4.2) and (4.9). For simplicity, we will focus on the error analysis for (4.2) only; the analysis can be easily modified to cover the WG-FEM scheme (4.9) without any difficulty.

First of all, let us derive an error equation for the weak Galerkin finite element solution.

Lemma 6.1. Let $u$ and $u_{h}=\left\{u_{0}, u_{b}, \mathbf{u}_{g}\right\} \in V_{h}$ be the solution of (1.1)-(1.3) and (4.2), respectively. Denote by

$$
e_{h}=Q_{h} u-u_{h}
$$

the error function between the $L^{2}$ projection of $u$ and its weak Galerkin finite element approximation. Then the error function $e_{h}$ satisfies the following equation

$$
\begin{aligned}
\left(\Delta_{w} e_{h}, \Delta_{w} v\right)_{h}+s\left(e_{h}, v\right) & =\sum_{T \in \mathcal{T}_{h}}\left\langle\Delta u-\mathbb{Q}_{h} \Delta u,\left(\nabla v_{0}-\mathbf{v}_{g}\right) \cdot \mathbf{n}\right\rangle_{\partial T} \\
& -\sum_{T \in \mathcal{T}_{h}}\left\langle\nabla\left(\Delta u-\mathbb{Q}_{h} \Delta u\right) \cdot \mathbf{n}, v_{0}-v_{b}\right\rangle_{\partial T}+s\left(Q_{h} u, v\right)
\end{aligned}
$$

for all $v \in V_{h}^{0}$.

Proof. Using (3.3), the integration by parts, and the fact that $\Delta_{w} Q_{h} u=\mathbb{Q}_{h}(\Delta u)$ we obtain

$$
\begin{aligned}
& \left(\Delta_{w} Q_{h} u, \Delta_{w} v\right)_{T} \\
= & \left(v_{0}, \Delta\left(\Delta_{w} Q_{h} u\right)\right)_{T}+\left\langle\mathbf{v}_{g} \cdot \mathbf{n}, \Delta_{w} Q_{h} u\right\rangle_{\partial T}-\left\langle v_{b}, \nabla\left(\Delta_{w} Q_{h} u\right) \cdot \mathbf{n}\right\rangle_{\partial T} \\
= & \left(\Delta v_{0}, \Delta_{w} Q_{h} u\right)_{T}+\left\langle v_{0}, \nabla\left(\Delta_{w} Q_{h} u\right) \cdot \mathbf{n}\right\rangle_{\partial T}-\left\langle\nabla v_{0} \cdot \mathbf{n}, \Delta_{w} Q_{h} u\right\rangle_{\partial T} \\
& +\left\langle\mathbf{v}_{g} \cdot \mathbf{n}, \Delta_{w} Q_{h} u\right\rangle_{\partial T}-\left\langle v_{b}, \nabla\left(\Delta_{w} Q_{h} u\right) \cdot \mathbf{n}\right\rangle_{\partial T} \\
= & \left(\Delta v_{0}, \Delta_{w} Q_{h} u\right)_{T}+\left\langle v_{0}-v_{b}, \nabla\left(\Delta_{w} Q_{h} u\right) \cdot \mathbf{n}\right\rangle_{\partial T}-\left\langle\left(\nabla v_{0}-\mathbf{v}_{g}\right) \cdot \mathbf{n}, \Delta_{w} Q_{h} u\right\rangle_{\partial T} \\
= & \left(\Delta v_{0}, \mathbb{Q}_{h} \Delta u\right)_{T}+\left\langle v_{0}-v_{b}, \nabla\left(\mathbb{Q}_{h} \Delta u\right) \cdot \mathbf{n}\right\rangle_{\partial T}-\left\langle\left(\nabla v_{0}-\mathbf{v}_{g}\right) \cdot \mathbf{n}, \mathbb{Q}_{h} \Delta u\right\rangle_{\partial T} \\
= & \left(\Delta u, \Delta v_{0}\right)_{T}+\left\langle v_{0}-v_{b}, \nabla\left(\mathbb{Q}_{h} \Delta u\right) \cdot \mathbf{n}\right\rangle_{\partial T}-\left\langle\left(\nabla v_{0}-\mathbf{v}_{g}\right) \cdot \mathbf{n}, \mathbb{Q}_{h} \Delta u\right\rangle_{\partial T},
\end{aligned}
$$

which implies that

$$
\begin{aligned}
\left(\Delta u, \Delta v_{0}\right)_{T} & =\left(\Delta_{w} Q_{h} u, \Delta_{w} v\right)_{T}-\left\langle v_{0}-v_{b}, \nabla\left(\mathbb{Q}_{h} \Delta u\right) \cdot \mathbf{n}\right\rangle_{\partial T} \\
& +\left\langle\left(\nabla v_{0}-\mathbf{v}_{g}\right) \cdot \mathbf{n}, \mathbb{Q}_{h} \Delta u\right\rangle_{\partial T} .
\end{aligned}
$$

Next, it follows from the integration by parts that

$$
\left(\Delta u, \Delta v_{0}\right)_{T}=\left(\Delta^{2} u, v_{0}\right)_{T}+\left\langle\Delta u, \nabla v_{0} \cdot \mathbf{n}\right\rangle_{\partial T}-\left\langle\nabla(\Delta u) \cdot \mathbf{n}, v_{0}\right\rangle_{\partial T} .
$$


Summing over all $T$ and then using the identity $\left(\Delta^{2} u, v_{0}\right)=\left(f, v_{0}\right)$ we arrive at $\sum_{T \in \mathcal{T}_{h}}\left(\Delta u, \Delta v_{0}\right)_{T}=\left(f, v_{0}\right)+\sum_{T \in \mathcal{T}_{h}}\left\langle\Delta u,\left(\nabla v_{0}-\mathbf{v}_{g}\right) \cdot \mathbf{n}\right\rangle_{\partial T}-\sum_{T \in \mathcal{T}_{h}}\left\langle\nabla(\Delta u) \cdot \mathbf{n}, v_{0}-v_{b}\right\rangle_{\partial T}$,

where we have used the fact that $\mathbf{v}_{g} \cdot \mathbf{n}$ and $v_{b}$ vanishes on the boundary of the domain. Combining the above equation with (6.2) leads to

$$
\begin{aligned}
\left(\Delta_{w} Q_{h} u, \Delta_{w} v\right)_{h} & =\left(f, v_{0}\right)+\sum_{T \in \mathcal{T}_{h}}\left\langle\Delta u-\mathbb{Q}_{h} \Delta u,\left(\nabla v_{0}-\mathbf{v}_{g}\right) \cdot \mathbf{n}\right\rangle_{\partial T} \\
& -\sum_{T \in \mathcal{T}_{h}}\left\langle\nabla\left(\Delta u-\mathbb{Q}_{h} \Delta u\right) \cdot \mathbf{n}, v_{0}-v_{b}\right\rangle_{\partial T} .
\end{aligned}
$$

Adding $s\left(Q_{h} u, v\right)$ to both sides of the above equation gives

$$
\begin{aligned}
\left(\Delta_{w} Q_{h} u, \Delta_{w} v\right)_{h}+s\left(Q_{h} u, v\right) & =\left(f, v_{0}\right)+\sum_{T \in \mathcal{T}_{h}}\left\langle\Delta u-\mathbb{Q}_{h} \Delta u,\left(\nabla v_{0}-\mathbf{v}_{g}\right) \cdot \mathbf{n}\right\rangle_{\partial T} \\
& -\sum_{T \in \mathcal{T}_{h}}\left\langle\nabla\left(\Delta u-\mathbb{Q}_{h} \Delta u\right) \cdot \mathbf{n}, v_{0}-v_{b}\right\rangle_{\partial T}+s\left(Q_{h} u, v\right) .
\end{aligned}
$$

Subtracting (4.2) from (6.3) yields the following error equation

$$
\begin{aligned}
\left(\Delta_{w} e_{h}, \Delta_{w} v\right)_{h}+s\left(e_{h}, v\right) & =\sum_{T \in \mathcal{T}_{h}}\left\langle\Delta u-\mathbb{Q}_{h} \Delta u,\left(\nabla v_{0}-\mathbf{v}_{g}\right) \cdot \mathbf{n}\right\rangle_{\partial T} \\
& -\sum_{T \in \mathcal{T}_{h}}\left\langle\nabla\left(\Delta u-\mathbb{Q}_{h} \Delta u\right) \cdot \mathbf{n}, v_{0}-v_{b}\right\rangle_{\partial T}+s\left(Q_{h} u, v\right)
\end{aligned}
$$

for all $v \in V_{h}^{0}$. This completes the derivation of (6.1).

The following is an estimate for the error function $e_{h}$ in the trip-bar norm which is essentially an $H^{2}$-norm in $V_{h}^{0}$.

THEOREM 6.2. Let $u_{h} \in V_{h}$ be the weak Galerkin finite element solution arising from (4.2) with finite element functions of order $k \geq 2$. Assume that the exact solution of (1.1)-(1.3) is sufficiently regular such that $u \in H^{\max \{k+1,4\}}(\Omega)$. Then, there exists a constant $C$ such that

$$
\left\|u_{h}-Q_{h} u\right\| \leq C h^{k-1}\left(\|u\|_{k+1}+h \delta_{k, 2}\|u\|_{4}\right) .
$$

In other words, we have the optimal order of convergence in the $H^{2}$ norm.

Proof. By letting $v=e_{h}$ in the error equation (6.1), we obtain the following identity

$$
\begin{aligned}
\left\|e_{h}\right\| & =\sum_{T \in \mathcal{T}_{h}}\left\langle\Delta u-\mathbb{Q}_{h} \Delta u,\left(\nabla e_{0}-\mathbf{e}_{g}\right) \cdot \mathbf{n}\right\rangle_{\partial T} \\
& -\sum_{T \in \mathcal{T}_{h}}\left\langle\nabla\left(\Delta u-\mathbb{Q}_{h} \Delta u\right) \cdot \mathbf{n}, e_{0}-e_{b}\right\rangle_{\partial T} \\
& +\sum_{T \in \mathcal{T}_{h}} h_{T}^{-1}\left\langle\nabla Q_{0} u-\mathbf{Q}_{g} \nabla u, \nabla e_{0}-\mathbf{e}_{g}\right\rangle_{\partial T} \\
& +\sum_{T \in \mathcal{T}_{h}} h_{T}^{-3}\left\langle Q_{0} u-Q_{b} u, e_{0}-e_{b}\right\rangle_{\partial T} .
\end{aligned}
$$


Now using the Cauchy-Schwarz inequality and the estimates (5.4) and (5.5) of Lemma 5.3 with $m=k$ one arrives at

$$
\begin{aligned}
& \left|\sum_{T \in \mathcal{T}_{h}}\left\langle\Delta u-\mathbb{Q}_{h} \Delta u,\left(\nabla e_{0}-\mathbf{e}_{g}\right) \cdot \mathbf{n}\right\rangle_{\partial T}\right| \\
\leq & \left(\sum_{T \in \mathcal{T}_{h}} h_{T}\left\|\Delta u-\mathbb{Q}_{h} \Delta u\right\|_{\partial T}^{2}\right)^{\frac{1}{2}}\left(\sum_{T \in \mathcal{T}_{h}} h_{T}^{-1}\left\|\nabla e_{0}-\mathbf{e}_{g}\right\|_{\partial T}^{2}\right)^{\frac{1}{2}} \\
\leq & C h^{k-1}\|u\|_{k+1}\left\|e_{h}\right\|
\end{aligned}
$$

and

$$
\begin{aligned}
& \left|\sum_{T \in \mathcal{T}_{h}}\left\langle\nabla\left(\Delta u-\mathbb{Q}_{h} \Delta u\right) \cdot \mathbf{n}, e_{0}-e_{b}\right\rangle_{\partial T}\right| \\
\leq & \left(\sum_{T \in \mathcal{T}_{h}} h_{T}^{3}\left\|\nabla\left(\Delta u-\mathbb{Q}_{h} \Delta u\right)\right\|_{\partial T}^{2}\right)^{\frac{1}{2}}\left(\sum_{T \in \mathcal{T}_{h}} h_{T}^{-3}\left\|e_{0}-e_{b}\right\|_{\partial T}^{2}\right)^{\frac{1}{2}} \\
\leq & C h^{k-1}\left(\|u\|_{k+1}+h \delta_{k, 2}\|u\|_{4}\right)\left\|e_{h}\right\| .
\end{aligned}
$$

Similarly, it follows from the Cauchy-Schwarz and the estimates (5.6) and (5.7) that

$$
\left|\sum_{T \in \mathcal{T}_{h}} h_{T}^{-1}\left\langle\nabla Q_{0} u-\mathbf{Q}_{g} \nabla u, \nabla e_{0}-\mathbf{e}_{g}\right\rangle_{\partial T}\right| \leq C h^{k-1}\|u\|_{k+1}\left\|e_{h}\right\|
$$

and

$$
\left|\sum_{T \in \mathcal{T}_{h}} h_{T}^{-3}\left\langle Q_{0} u-Q_{b} u, e_{0}-e_{b}\right\rangle_{\partial T}\right| \leq C h^{k-1}\|u\|_{k+1}\left\|e_{h}\right\| .
$$

Substituting (6.6)-(6.9) into (6.5) yields

$$
\left\|e_{h}\right\|^{2} \leq C h^{k-1}\left(\|u\|_{k+1}+h \delta_{k, 2}\|u\|_{4}\right)\left\|e_{h}\right\|,
$$

which implies (6.4). This completes the proof of the theorem.

7. An Error Estimate in $L^{2}$. This section shall establish an estimate for the first component of the error function $e_{h}$ in the standard $L^{2}$ norm. To this end, we consider the following dual problem

$$
\begin{aligned}
\Delta^{2} w & =e_{0} & & \text { in } \Omega, \\
w & =0, & & \text { on } \partial \Omega, \\
\nabla w \cdot \mathbf{n} & =0 & & \text { on } \partial \Omega .
\end{aligned}
$$

Assume that the above dual problem has the following regularity estimate

$$
\|w\|_{4} \leq C\left\|e_{0}\right\| .
$$

THEOREM 7.1. Let $u_{h} \in V_{h}$ be the weak Galerkin finite element solution arising from (4.2) with finite element functions of order $k \geq 2$. Let $t_{0}=\min (k, 3)$. Assume 
that the exact solution of (1.1)-(1.3) is sufficiently regular such that $u \in H^{k+1}(\Omega)$ and the dual problem (7.1)-(7.3) has the $H^{4}$ regularity. Then, there exists a constant $C$ such that

$$
\left\|Q_{0} u-u_{0}\right\| \leq C h^{k+t_{0}-2}\left(\|u\|_{k+1}+h \delta_{k, 2}\|u\|_{4}\right) .
$$

In other words, for quadratic elements (i.e., $k=2$ ) we have a sub-optimal order of convergence given by $\left\|Q_{0} u-u_{0}\right\|=\mathcal{O}\left(h^{2}\right)$. But for cubic or higher order of elements, we have the optimal order of convergence $\left\|Q_{0} u-u_{0}\right\|=\mathcal{O}\left(h^{k+1}\right)$.

Proof. Testing (7.1) with the error function $e_{0}$ on each element and then using integration by parts to obtain

$$
\begin{aligned}
\left\|e_{0}\right\|^{2} & =\left(\Delta^{2} w, e_{0}\right) \\
& =\sum_{T \in \mathcal{T}_{h}}\left(\Delta w, \Delta e_{0}\right)_{T}+\sum_{T \in \mathcal{T}_{h}}\left\langle\nabla(\Delta w) \cdot \mathbf{n}, e_{0}\right\rangle_{\partial T}-\sum_{T \in \mathcal{T}_{h}}\left\langle\Delta w, \nabla e_{0} \cdot \mathbf{n}\right\rangle_{\partial T} \\
& =\sum_{T \in \mathcal{T}_{h}}\left(\Delta w, \Delta e_{0}\right)_{T}+\sum_{T \in \mathcal{T}_{h}}\left\langle\nabla(\Delta w) \cdot \mathbf{n}, e_{0}-e_{b}\right\rangle_{\partial T}-\sum_{T \in \mathcal{T}_{h}}\left\langle\Delta w,\left(\nabla e_{0}-\mathbf{e}_{g}\right) \cdot \mathbf{n}\right\rangle_{\partial T},
\end{aligned}
$$

where we have used the fact that $e_{b}$ and $\mathbf{e}_{g} \cdot \mathbf{n}$ vanishes on the boundary of $\Omega$. Using (6.2) with $w$ in the place of $u$, we can rewrite the above identity as follows

$$
\begin{aligned}
\left\|e_{0}\right\|^{2}= & \left(\Delta_{w} Q_{h} w, \Delta_{w} e_{h}\right)_{h}+\sum_{T \in \mathcal{T}_{h}}\left\langle\left(\nabla(\Delta w)-\nabla\left(\mathbb{Q}_{h} \Delta w\right)\right) \cdot \mathbf{n}, e_{0}-e_{b}\right\rangle_{\partial T} \\
& -\sum_{T \in \mathcal{T}_{h}}\left\langle\Delta w-\mathbb{Q}_{h} \Delta w,\left(\nabla e_{0}-\mathbf{e}_{g}\right) \cdot \mathbf{n}\right\rangle_{\partial T} .
\end{aligned}
$$

Next, we have from the error equation (6.1) that

$$
\begin{aligned}
\left(\Delta_{w} Q_{h} w, \Delta_{w} e_{h}\right)_{h} & =\sum_{T \in \mathcal{T}_{h}}\left\langle\Delta u-\mathbb{Q}_{h} \Delta u,\left(\nabla Q_{0} w-\mathbf{Q}_{g} \nabla w\right) \cdot \mathbf{n}\right\rangle_{\partial T} \\
& -\sum_{T \in \mathcal{T}_{h}}\left\langle\nabla\left(\Delta u-\mathbb{Q}_{h} \Delta u\right) \cdot \mathbf{n}, Q_{0} w-Q_{b} w\right\rangle_{\partial T} \\
& -s\left(e_{h}, Q_{h} w\right)+s\left(Q_{h} u, Q_{h} w\right) .
\end{aligned}
$$

Combining the above two equations we obtain

$$
\begin{aligned}
\left\|e_{0}\right\|^{2} & =\sum_{T \in \mathcal{T}_{h}}\left\langle\left(\nabla(\Delta w)-\nabla\left(\mathbb{Q}_{h} \Delta w\right)\right) \cdot \mathbf{n}, e_{0}-e_{b}\right\rangle_{\partial T} \\
& -\sum_{T \in \mathcal{T}_{h}}\left(\Delta w-\mathbb{Q}_{h} \Delta w,\left(\nabla e_{0}-\mathbf{e}_{g}\right) \cdot \mathbf{n}\right\rangle_{\partial T}-s\left(e_{h}, Q_{h} w\right) \\
& +\sum_{T \in \mathcal{T}_{h}}\left\langle\Delta u-\mathbb{Q}_{h} \Delta u,\left(\nabla Q_{0} w-\mathbf{Q}_{g} \nabla w\right) \cdot \mathbf{n}\right\rangle_{\partial T} \\
& -\sum_{T \in \mathcal{T}_{h}}\left\langle\nabla\left(\Delta u-\mathbb{Q}_{h} \Delta u\right) \cdot \mathbf{n}, Q_{0} w-Q_{b} w\right\rangle_{\partial T}+s\left(Q_{h} u, Q_{h} w\right) .
\end{aligned}
$$

Each of the six terms on the right-hand side of (7.6) can be bounded by using the Cauchy-Schwarz inequality and Lemma 5.3 as follows. 
For the first term, it follows from the estimate (5.5) and the fact $t_{0}=\min (3, k) \leq 3$ that

$$
\begin{aligned}
& \left|\sum_{T \in \mathcal{T}_{h}}\left\langle\nabla\left(\Delta w-\mathbb{Q}_{h} \Delta w\right) \cdot \mathbf{n}, e_{0}-e_{b}\right\rangle_{\partial T}\right| \\
\leq & \left(\sum_{T \in \mathcal{T}_{h}} h_{T}^{3}\left\|\nabla\left(\Delta w-\mathbb{Q}_{h} \Delta w\right)\right\|_{\partial T}^{2}\right)^{\frac{1}{2}}\left(\sum_{T \in \mathcal{T}_{h}} h_{T}^{-3}\left\|e_{0}-e_{b}\right\|_{\partial T}^{2}\right)^{\frac{1}{2}} \\
\leq C h^{t_{0}-1}\left(\|w\|_{t_{0}+1}+h \delta_{t_{0}, 2}\|w\|_{4}\right)\left\|e_{h}\right\| & \leq C h^{t_{0}-1}\|w\|_{4}\left\|e_{h}\right\| .
\end{aligned}
$$

As to the second term, we use the Cauchy-Schwarz inequality and the estimate (5.4) with $m=t_{0}$ to obtain

$$
\begin{aligned}
& \left|\sum_{T \in \mathcal{T}_{h}}\left\langle\Delta w-\mathbb{Q}_{h} \Delta w,\left(\nabla e_{0}-\mathbf{e}_{g}\right) \cdot \mathbf{n}\right\rangle_{\partial T}\right| \\
\leq & \left(\sum_{T \in \mathcal{T}_{h}} h_{T}\left\|\Delta w-\mathbb{Q}_{h} \Delta w\right\|_{\partial T}^{2}\right)^{\frac{1}{2}}\left(\sum_{T \in \mathcal{T}_{h}} h_{T}^{-1}\left\|\nabla e_{0}-\mathbf{e}_{g}\right\|_{\partial T}^{2}\right)^{\frac{1}{2}} \\
\leq C h^{t_{0}-1}\|w\|_{t_{0}+1}\left\|e_{h}\right\| & \leq C h^{t_{0}-1}\|w\|_{4}\left\|e_{h}\right\| .
\end{aligned}
$$

Analogously, for the third term, we have

$$
\left|s\left(e_{h}, Q_{h} w\right)\right| \leq C h^{t_{0}-1}\|w\|_{4}\left\|e_{h}\right\| .
$$

The fourth term can be bounded by using Lemma 5.2 as follows

$$
\begin{aligned}
& \left|\sum_{T \in \mathcal{T}_{h}}\left\langle\Delta u-\mathbb{Q}_{h} \Delta u,\left(\nabla Q_{0} w-\mathbf{Q}_{g} \nabla w\right) \cdot \mathbf{n}\right\rangle_{\partial T}\right| \\
\leq & \left(\sum_{T \in \mathcal{T}_{h}} h\left\|\Delta u-\mathbb{Q}_{h} \Delta u\right\|_{\partial T}^{2}\right)^{\frac{1}{2}}\left(\sum_{T \in \mathcal{T}_{h}} h^{-1}\left\|\nabla Q_{0} w-\nabla w\right\|_{\partial T}^{2}\right)^{\frac{1}{2}} \\
\leq & C h^{k-1}\|u\|_{k+1} h^{t_{0}-1}\|w\|_{t_{0}+1} \\
\leq & C h^{k+t_{0}-2}\|u\|_{k+1}\|w\|_{4} .
\end{aligned}
$$

As to the fifth term, we again use the Cauchy-Schwarz inequality and Lemma 5.3 to obtain

$$
\begin{aligned}
& \left|\sum_{T \in \mathcal{T}_{h}}\left\langle\nabla\left(\Delta u-\mathbb{Q}_{h} \Delta u\right) \cdot \mathbf{n}, Q_{0} w-Q_{b} w\right\rangle_{\partial T}\right| \\
& \leq\left(\sum_{T \in \mathcal{T}_{h}} h_{T}^{3}\left\|\nabla\left(\Delta u-\mathbb{Q}_{h} \Delta u\right)\right\|_{\partial T}^{2}\right)^{\frac{1}{2}}\left(\sum_{T \in \mathcal{T}_{h}} h_{T}^{-3}\left\|Q_{0} w-w\right\|_{\partial T}^{2}\right)^{\frac{1}{2}} \\
& \leq C h^{k-1}\left(\|u\|_{k+1}+h \delta_{k, 2}\|u\|_{4}\right) h^{t_{0}-1}\|w\|_{t_{0}+1} \\
& \leq C h^{k+t_{0}-2}\left(\|u\|_{k+1}+h \delta_{k, 2}\|u\|_{4}\right)\|w\|_{4}
\end{aligned}
$$


The last term on the right-hand side of (7.6) can be estimated as follows.

$$
\begin{aligned}
& \left|s\left(Q_{h} u, Q_{h} w\right)\right| \\
\leq & \left|\sum_{T \in \mathcal{T}_{h}} h_{T}^{-1}\left\langle\nabla Q_{0} u-\mathbf{Q}_{g} \nabla u, \nabla Q_{0} w-\mathbf{Q}_{g} \nabla w\right\rangle_{\partial T}\right| \\
+ & \left|\sum_{T \in \mathcal{T}_{h}} h_{T}^{-3}\left\langle Q_{0} u-Q_{b} u, Q_{0} w-Q_{b} w\right\rangle_{\partial T}\right| \\
\leq & \left(\sum_{T \in \mathcal{T}_{h}} h_{T}^{-1}\left\|\nabla Q_{0} u-\nabla u\right\|_{\partial T}^{2}\right)^{\frac{1}{2}}\left(\sum_{T \in \mathcal{T}_{h}} h_{T}^{-1}\left\|\nabla Q_{0} w-\nabla w\right\|_{\partial T}^{2}\right)^{\frac{1}{2}} \\
+ & \left(\sum_{T \in \mathcal{T}_{h}} h_{T}^{-3}\left\|Q_{0} u-u\right\|_{\partial T}^{2}\right)^{\frac{1}{2}}\left(\sum_{T \in \mathcal{T}_{h}} h_{T}^{-3}\left\|Q_{0} w-w\right\|_{\partial T}^{2}\right)^{\frac{1}{2}} \\
\leq & C h^{k-1}\|u\|_{k+1} h^{t_{0}-1}\|w\|_{t_{0}+1} \\
\leq & C h^{k+t_{0}-2}\|u\|_{k+1}\|w\|_{4} .
\end{aligned}
$$

Substituting the estimates (7.7)-(7.12) into (7.6) yields

$$
\left\|e_{0}\right\|^{2} \leq C h^{t_{0}-1}\left\|e_{h}\right\|\|w\|_{4}+C h^{k+t_{0}-2}\left(\|u\|_{k+1}+h \delta_{k, 2}\|u\|_{4}\right)\|w\|_{4} .
$$

Using the regularity estimate (7.4) we arrive at

$$
\left\|e_{0}\right\| \leq C h^{t_{0}-1}\left\|e_{h}\right\|+C h^{k+t_{0}-2}\left(\|u\|_{k+1}+h \delta_{k, 2}\|u\|_{4}\right) .
$$

Finally, by combining the above estimate with the $H^{2}$ error estimate (6.4) we obtain the desired $L^{2}$ error estimate (7.5).

8. Numerical Results. Our numerical experiments are conducted for the weak Galerkin finite element scheme (4.9) by using the following finite element space

$$
\tilde{V}_{h}=\left\{v=\left\{v_{0}, v_{b}, v_{g} \mathbf{n}_{e}\right\}, v_{0} \in P_{2}(T), v_{b} \in P_{2}(e), v_{g} \in P_{1}(e), T \in \mathcal{T}_{h}, e \in \mathcal{E}_{h}\right\} .
$$

For any given $v=\left\{v_{0}, v_{b}, v_{g} \mathbf{n}_{e}\right\} \in \tilde{V}_{h}$, the discrete weak Laplacian, $\Delta_{w} v$, is computed locally on each element $T$ as a function in $P_{0}(T)$ by solving the following equation

$$
\left(\Delta_{w} v, \psi\right)_{T}=\left(v_{0}, \Delta \psi\right)_{T}+\left\langle v_{g} \mathbf{n}_{e} \cdot \mathbf{n}, \psi\right\rangle_{\partial T}-\left\langle v_{b}, \nabla \psi \cdot \mathbf{n}\right\rangle_{\partial T},
$$

for all $\psi \in P_{0}(T)$. Since $\psi \in P_{0}(T)$, the above equation can be simplified as

$$
\left(\Delta_{w} v, \psi\right)_{T}=\left\langle v_{g} \mathbf{n}_{e} \cdot \mathbf{n}, \psi\right\rangle_{\partial T} .
$$

The error for the WG-FEM solution is measured in two norms defined as follows:

$$
\begin{array}{rlrl}
\left\|v_{h}\right\|^{2}:= & \sum_{T \in \mathcal{T}_{h}}\left(\int_{T}\left|\Delta_{w} v_{h}\right|^{2} d x+h_{T}^{-1} \int_{\partial T}\left|\left(\nabla v_{0}\right) \cdot \mathbf{n}_{e}-v_{g}\right|^{2} d s\right. \\
& \left.+h_{T}^{-3} \int_{\partial T}\left(v_{0}-v_{b}\right)^{2} d s\right), & & \text { (A discrete } H^{2} \text {-norm), } \\
\left\|v_{h}\right\|^{2}:= & \sum_{T \in \mathcal{T}_{h}} \int_{T}\left|v_{0}\right|^{2} d x, & & \text { (Element-based } L^{2} \text {-norm). }
\end{array}
$$


8.1. Test Case 1. Here we consider the forth order problem that seeks an unknown function $u=u(x, y)$ satisfying

$$
\Delta^{2} u=f
$$

in the square domain $\Omega=(0,1)^{2}$ with homogeneous Dirichlet and Neumann boundary conditions. The exact solution is given by $u=x^{2}(1-x)^{2} y^{2}(1-y)^{2}$, and the function $f=f(x, y)$ is computed to match the exact solution. Uniform triangular meshes are used and they are constructed as follows: (1) partition the domain into $n \times n$ sub-rectangles; (2) divide each square element into two triangles by the diagonal line with a negative slope. The mesh size is denoted by $h=1 / n$. Table 8.1 shows that the convergence rates for the WG-FEM solution in the $H^{2}$ and $L^{2}$ norms are of order $O(h)$ and $O\left(h^{2}\right)$, respectively.

TABLE 8.1

Test Case 1: Numerical error and convergence rates in $H^{2}$ and $L^{2}$.

\begin{tabular}{|c||c|c|c|c|}
\hline$h$ & $\left\|u_{h}-Q_{h} u\right\|$ & order & $\left\|u_{0}-Q_{0} u\right\|$ & order \\
\hline \hline $2.5000 \mathrm{e}-01$ & $2.5683 \mathrm{e}-01$ & & $3.3304 \mathrm{e}-02$ & \\
\hline $1.2500 \mathrm{e}-01$ & $1.3540 \mathrm{e}-01$ & $9.2359 \mathrm{e}-01$ & $9.1046 \mathrm{e}-03$ & 1.8710 \\
\hline $6.2500 \mathrm{e}-02$ & $7.2378 \mathrm{e}-02$ & $9.0360 \mathrm{e}-01$ & $2.6049 \mathrm{e}-03$ & 1.8054 \\
\hline $3.1250 \mathrm{e}-02$ & $3.8275 \mathrm{e}-02$ & $9.1915 \mathrm{e}-01$ & $7.3257 \mathrm{e}-04$ & 1.8302 \\
\hline $1.5625 \mathrm{e}-02$ & $1.9687 \mathrm{e}-02$ & $9.5916 \mathrm{e}-01$ & $1.9461 \mathrm{e}-04$ & 1.9124 \\
\hline $7.8125 \mathrm{e}-03$ & $9.9457 \mathrm{e}-03$ & $9.8510 \mathrm{e}-01$ & $4.9762 \mathrm{e}-05$ & 1.9675 \\
\hline
\end{tabular}

8.2. Test Case 2. Here we solve the biharmonic equation on the domain of unit square with nonhomogeneous Dirichlet and Neumann boundary conditions. The exact solution is given by $u=\sin (\pi x) \sin (\pi y)$, and the function $f=f(x, y)$ is computed accordingly.

The mesh triangulations are constructed in the same way as in the test case 1 . The numerical results are presented in Table 8.2 which confirm the theory developed in earlier sections.

TABLE 8.2

Test Case 2: Numerical error and convergence rates for the biharmonic equation with nonhomogeneous boundary conditions.

\begin{tabular}{|c||c|c|c|c|}
\hline$h$ & $\left\|u_{h}-Q_{h} u\right\|$ & order & $\left\|u_{0}-Q_{0} u\right\|$ & order \\
\hline \hline $2.5000 \mathrm{e}-01$ & $2.4536 \mathrm{e}+01$ & & $3.1862 \mathrm{e}+00$ & \\
\hline $1.2500 \mathrm{e}-01$ & $1.2794 \mathrm{e}+01$ & $9.3943 \mathrm{e}-01$ & $8.5298 \mathrm{e}-01$ & 1.9013 \\
\hline $6.2500 \mathrm{e}-02$ & $6.7243 \mathrm{e}+00$ & $9.2801 \mathrm{e}-01$ & $2.3439 \mathrm{e}-01$ & 1.8636 \\
\hline $3.1250 \mathrm{e}-02$ & $3.4811 \mathrm{e}+00$ & $9.4984 \mathrm{e}-01$ & $6.2578 \mathrm{e}-02$ & 1.9052 \\
\hline $1.5625 \mathrm{e}-02$ & $1.7657 \mathrm{e}+00$ & $9.7930 \mathrm{e}-01$ & $1.6066 \mathrm{e}-02$ & 1.9616 \\
\hline $7.8125 \mathrm{e}-03$ & $8.8709 \mathrm{e}-01$ & $9.9309 \mathrm{e}-01$ & $4.0534 \mathrm{e}-03$ & 1.9868 \\
\hline
\end{tabular}

More numerical experiments should be conducted for the WG-FEM schemes (4.2) and (4.9). In particular, it would be interesting to see results for WG approximations of high order elements. It is also important to hybridize (4.2) and (4.9) so that unknowns related to $v_{0}$ can be eliminated locally on each element. 
Appendix A. The goal of this Appendix is to establish some fundamental estimates useful in the error estimate for general weak Galerkin finite element methods. First, we derive a trace inequality for functions defined on the finite element partition $\mathcal{T}_{h}$ with properties as specified in [12]. For completeness, we review the shape regularity assumption in the following subsection.

A.1. Domain Partition and Shape Regularity. Let $\mathcal{T}_{h}$ be a partition of the domain $\Omega$ consisting of polygons in two dimensions or polyhedra in three dimensions satisfying a set of conditions to be specified. Denote by $\mathcal{E}_{h}$ the set of all edges or flat faces in $\mathcal{T}_{h}$, and let $\mathcal{E}_{h}^{0}=\mathcal{E}_{h} \backslash \partial \Omega$ be the set of all interior edges or flat faces. For every element $T \in \mathcal{T}_{h}$, we denote by $|T|$ the area or volume of $T$ and by $h_{T}$ its diameter. Similarly, we denote by $|e|$ the length or area of $e$ and by $h_{e}$ the diameter of edge or flat face $e \in \mathcal{E}_{h}$. We also set as usual the mesh size of $\mathcal{T}_{h}$ by

$$
h=\max _{T \in \mathcal{T}_{h}} h_{T} .
$$

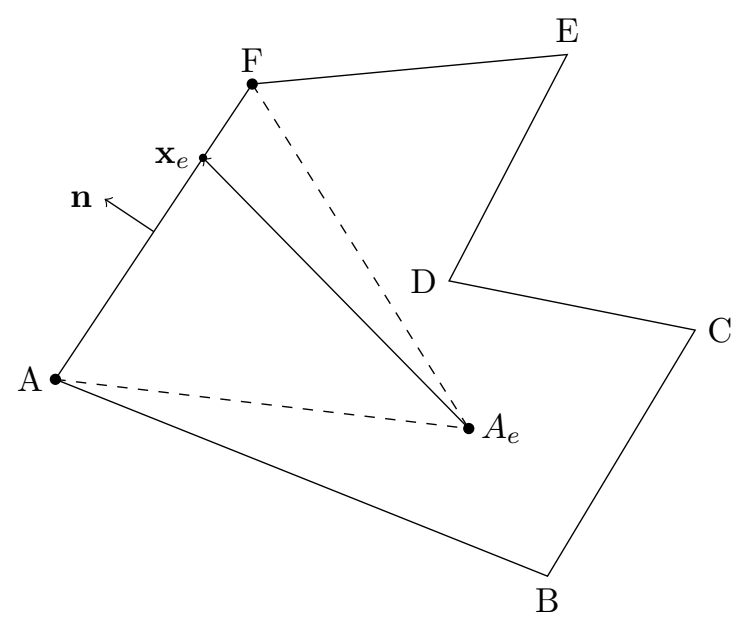

FIG. A.1. Depiction of a shape-regular polygonal element ABCDEFA.

All the elements of $\mathcal{T}_{h}$ are assumed to be closed and simply connected polygons or polyhedra, see Fig. A.1. The partition $\mathcal{T}_{h}$ is said to be shape regular if the following properties are satisfied.

A1: Assume that there exist two positive constants $\varrho_{v}$ and $\varrho_{e}$ such that for every element $T \in \mathcal{T}_{h}$ we have

$$
\varrho_{v} h_{T}^{d} \leq|T|, \quad \varrho_{e} h_{e}^{d-1} \leq|e|
$$

for all edges or flat faces of $T$.

A2: Assume that there exists a positive constant $\kappa$ such that for every element $T \in \mathcal{T}_{h}$ we have

$$
\kappa h_{T} \leq h_{e}
$$

for all edges or flat faces $e$ of $T$. 
A3: Assume that the mesh edges or faces are flat. We further assume that for every $T \in \mathcal{T}_{h}$, and for every edge/face $e \in \partial T$, there exists a pyramid $P\left(e, T, A_{e}\right)$ contained in $T$ such that its base is identical with $e$, its apex is $A_{e} \in T$, and its height is proportional to $h_{T}$ with a proportionality constant $\sigma_{e}$ bounded from below by a fixed positive number $\sigma^{*}$. In other words, the height of the pyramid is given by $\sigma_{e} h_{T}$ such that $\sigma_{e} \geq \sigma^{*}>0$. The pyramid is also assumed to stand up above the base $e$ in the sense that the angle between the vector $\mathbf{x}_{e}-A_{e}$, for any $x_{e} \in e$, and the outward normal direction of $e$ is strictly acute by falling into an interval $\left[0, \theta_{0}\right]$ with $\theta_{0}<\frac{\pi}{2}$.

A4: Assume that each $T \in \mathcal{T}_{h}$ has a circumscribed simplex $S(T)$ that is shape regular and has a diameter $h_{S(T)}$ proportional to the diameter of $T$; i.e., $h_{S(T)} \leq \gamma_{*} h_{T}$ with a constant $\gamma_{*}$ independent of $T$. Furthermore, assume that each circumscribed simplex $S(T)$ intersects with only a fixed and small number of such simplices for all other elements $T \in \mathcal{T}_{h}$.

A.2. A Trace Inequality. The following is trace inequality concerning functions in the Sobolev space $W^{1, p}$.

Lemma A.1 (Trace Inequality). Let $\mathcal{T}_{h}$ be a partition of the domain $\Omega$ into polygons in $2 D$ or polyhedra in $3 D$. Assume that the partition $\mathcal{T}_{h}$ satisfies the assumptions (A1), (A2), and (A3) as specified in Section [12]. Let $p>1$ be any real number. Then, there exists a constant $C$ such that for any $T \in \mathcal{T}_{h}$ and edge/face $e \in \partial T$, we have

$$
\|\theta\|_{L^{p}(e)}^{p} \leq C h_{T}^{-1}\left(\|\theta\|_{L^{p}(T)}^{p}+h_{T}^{p}\|\nabla \theta\|_{L^{p}(T)}^{p}\right),
$$

where $\theta \in W^{1, p}(T)$ is any function.

Proof. We shall provide a proof for the case of $e$ being a flat face and $\theta \in C^{1}(T)$. To this end, let the flat face $e$ be represented by the following parametric equation:

$$
\mathbf{x}_{e}=\phi(\xi, \eta):=\left(\phi_{1}(\xi, \eta), \phi_{2}(\xi, \eta), \phi_{3}(\xi, \eta)\right)
$$

for $(\xi, \eta) \in D \subset \mathbb{R}^{2}$. By Assumption A3, there exists a pyramid $P\left(e, T, A_{e}\right)$ with apex $A_{e}=\mathbf{x}_{*}:=\left(x_{1}^{*}, x_{2}^{*}, x_{3}^{*}\right)$ contained in the element $T$. This pyramid has the following parametric representation:

$$
\mathbf{x}(t, \xi, \eta)=(1-t) \phi(\xi, \eta)+t \mathbf{x}_{*}
$$

for $(t, \xi, \eta) \in[0,1] \times D$. For any given $\mathbf{x}_{e} \in e$, the line segment joining $\mathbf{x}_{e}$ and the apex $\mathbf{x}_{*}$ can be represented by

$$
\mathbf{x}(t)=\mathbf{x}_{e}+t\left(\mathbf{x}_{*}-\mathbf{x}_{e}\right) .
$$

From the fundamental theorem of Calculus, we have

$$
|\theta|^{p}\left(\mathbf{x}_{e}\right)-|\theta|^{p}(\mathbf{x}(t))=-\int_{0}^{t} \partial_{\tau}\left(|\theta|^{p}\left(\mathbf{x}_{e}+\tau \omega\right)\right) d \tau, \quad \omega=\mathbf{x}_{*}-\mathbf{x}_{e} .
$$

The above can be further rewritten as

$$
|\theta|^{p}\left(\mathbf{x}_{e}\right)-|\theta|^{p}(\mathbf{x}(t))=-p \int_{0}^{t}|\theta|^{p-1} \operatorname{sgn}(\theta)(\nabla \theta \cdot \omega) d \tau .
$$


Let $q=\frac{p}{p-1}$. It follows from the Cauchy-Schwarz inequality that for any $t \in\left[0, \frac{1}{2}\right]$ we have

$$
|\theta|^{p}\left(\mathbf{x}_{e}\right) \leq|\theta|^{p}(\mathbf{x}(t))+p\left(\int_{0}^{\frac{1}{2}}|\theta|^{p}|\omega| d \tau\right)^{\frac{1}{q}}\left(\int_{0}^{\frac{1}{2}}|\nabla \theta|^{p}|\omega|\right)^{\frac{1}{p}}
$$

Using the Young's inequality $a b \leq \varepsilon a^{p}+\varepsilon^{-\frac{q}{p}} b^{q}$ for any non-negative real numbers $a, b$, and $\varepsilon>0$, we arrive at

$$
|\theta|^{p}\left(\mathbf{x}_{e}\right) \leq|\theta|^{p}(\mathbf{x}(t))+p \varepsilon^{-\frac{q}{p}} \int_{0}^{\frac{1}{2}}|\theta|^{p}|\omega| d \tau+p \varepsilon \int_{0}^{\frac{1}{2}}|\nabla \theta|^{p}|\omega| d \tau .
$$

Now we integrate the above inequality over the flat face $e$ by using the parametric equation (A.4), yielding

$$
\begin{aligned}
\int_{D}|\theta|^{p}\left(\mathbf{x}_{e}\right)\left|\phi_{\xi} \times \phi_{\eta}\right| d \xi d \eta \leq & \int_{D}|\theta|^{p}(\mathbf{x}(t))\left|\phi_{\xi} \times \phi_{\eta}\right| d \xi d \eta \\
& +p \varepsilon^{-\frac{q}{p}} \int_{0}^{\frac{1}{2}} \int_{D}|\theta|^{p}|\omega|\left|\phi_{\xi} \times \phi_{\eta}\right| d \xi d \eta d \tau \\
& +p \varepsilon \int_{0}^{\frac{1}{2}} \int_{D}|\nabla \theta|^{p}|\omega|\left|\phi_{\xi} \times \phi_{\eta}\right| d \xi d \eta d \tau
\end{aligned}
$$

Observe that the integral of a function over the following prismatoid

$$
P_{\frac{1}{2}}:=\{\mathbf{x}(t, \xi, \eta): \quad(t, \xi, \eta) \in[0,1 / 2] \times D\}
$$

is given by

$$
\int_{P_{\frac{1}{2}}} f(\mathbf{x}) d \mathbf{x}=\int_{0}^{\frac{1}{2}} \int_{D} f(\mathbf{x}(\tau, \xi, \eta)) J(\tau, \xi, \eta) d \xi d \eta d \tau
$$

where $J(\tau, \xi, \eta)=(1-\tau)^{2}\left|\left(\phi_{\xi} \times \phi_{\eta}\right) \cdot \omega\right|$ is the Jacobian from the coordinate change. The vector $\phi_{\xi} \times \phi_{\eta}$ is normal to the face $e$, and $\omega=\mathbf{x}_{*}-\mathbf{x}_{e}$ is a vector from the base point $\mathbf{x}_{e}$ to the apex $\mathbf{x}_{*}$. The angle assumption (see Assumption A3 of Section ??) for the prism $P\left(e, T, A_{e}\right)$ indicates that the Jacobian satisfies the following relation

$$
J(\tau, \xi, \eta) \geq \frac{\mu_{0}}{4}\left|\phi_{\xi} \times \phi_{\eta}\right||\omega|, \quad \tau \in[0,1 / 2]
$$

for some fixed $\mu_{0} \in(0,1)$. Observe that the left-hand side of (A.6) is the surface integral of $|\theta|^{p}$ over the face $e$. Thus, substituting (A.8) into (A.6) and (A.7) yields

$$
\begin{aligned}
\int_{e}|\theta|^{p} d e \leq & \int_{D}|\theta|^{p}(\mathbf{x}(t))\left|\phi_{\xi} \times \phi_{\eta}\right| d \xi d \eta \\
& +4 p \mu_{0}^{-1} \varepsilon^{-\frac{q}{p}} \int_{P_{\frac{1}{2}}}|\theta|^{p} d \mathbf{x}+4 p \mu_{0}^{-1} \varepsilon \int_{P_{\frac{1}{2}}}|\nabla \theta|^{p} d \mathbf{x} .
\end{aligned}
$$

Now we integrate the above with respect to $t$ in the interval $\left[0, \frac{1}{2}\right]$ to obtain

$$
\begin{aligned}
\frac{1}{2} \int_{e}|\theta|^{p} d e \leq & \int_{0}^{\frac{1}{2}} \int_{D}|\theta|^{p}(\mathbf{x}(t))\left|\phi_{\xi} \times \phi_{\eta}\right| d \xi d \eta d t \\
& +2 p \mu_{0}^{-1} \varepsilon^{-\frac{q}{p}} \int_{P_{\frac{1}{2}}}|\theta|^{p} d \mathbf{x}+2 p \mu_{0}^{-1} \varepsilon \int_{P_{\frac{1}{2}}}|\nabla \theta|^{p} d \mathbf{x} .
\end{aligned}
$$


Again, by substituting (A.8) into the right-hand side of (A.9) we arrive at

$$
\begin{aligned}
\frac{1}{2} \int_{e}|\theta|^{p} d e \leq & 4 \mu_{0}^{-1}|\omega|^{-1} \int_{0}^{\frac{1}{2}} \int_{D}|\theta|^{p}(\mathbf{x}(t)) J(t, \xi, \eta) d \xi d \eta d t \\
& +2 p \mu_{0}^{-1} \varepsilon^{-\frac{q}{p}} \int_{P_{\frac{1}{2}}}|\theta|^{p} d \mathbf{x}+2 p \mu_{0}^{-1} \varepsilon \int_{P_{\frac{1}{2}}}|\nabla \theta|^{p} d \mathbf{x} .
\end{aligned}
$$

The first integral on the right-hand side of (A.10) is the integral of $|\theta|^{p}$ on the prismatoid $P_{\frac{1}{2}}$. It can be seen from the Assumption A3 that

$$
|\omega|^{-1} \leq \alpha_{*} h_{T}^{-1}
$$

for some positive constant $\alpha_{*}$. By taking $\varepsilon=h_{T}^{p-1}$, we have $\varepsilon^{-\frac{q}{p}}=h_{T}^{-1}$. It then follows from (A.10) and (A.11) that

$$
\int_{e}|\theta|^{p} d e \leq C h_{T}^{-1}\left(\int_{P_{\frac{1}{2}}}|\theta|^{p} d \mathbf{x}+h_{T}^{p} \int_{P_{\frac{1}{2}}}|\nabla \theta|^{p} d \mathbf{x}\right),
$$

which completes the proof of the Lemma. $\square$

A.3. A Domain Inverse Inequality. Next, we would like to establish an estimate for the $L^{p}$ norm of polynomial functions by their $L^{p}$ norm on a subdomain. To this end, we first derive a result of similar nature for general functions in $W^{1, p}$.

Lemma A.2. Let $K \subset \mathbb{R}^{d}$ be convex and $v \in W^{1, p}(K)$ with $p \geq 1$. Then,

$$
\|v\|_{L^{p}(K)}^{p} \leq \frac{2|K|}{|S|}\|v\|_{L^{p}(S)}^{p}+\left(\frac{2 p \omega_{d} \delta^{d+1}}{|S|}\right)^{p}\|\nabla v\|_{L^{p}(K)}^{p},
$$

where $\delta$ is the diameter of $K, S$ is any measurable subset of $K$, and $\omega_{d}=\frac{2 \pi^{d / 2}}{d \Gamma(d / 2)}$ is the volume of unit ball in $\mathbb{R}^{d}$.

Proof. Since $C^{1}(K)$ is dense in $W^{1, p}(K)$, it is sufficient to establish (A.12) for $v \in C^{1}(K)$. For any $\mathbf{x}, \mathbf{y} \in K$, we have

$$
|v|^{p}(\mathbf{x})=|v|^{p}(\mathbf{y})-\int_{0}^{|\mathbf{x}-\mathbf{y}|} \partial_{r}\left(|v|^{p}(\mathbf{x}+r \omega)\right) d r, \quad \omega=\frac{\mathbf{y}-\mathbf{x}}{|\mathbf{y}-\mathbf{x}|} .
$$

From the usual chain rule and the Cauchy-Schwarz inequality we obtain

$$
\begin{aligned}
|v|^{p}(\mathbf{x}) & =|v|^{p}(\mathbf{y})-p \int_{0}^{|\mathbf{x}-\mathbf{y}|}|v|^{p-1} \operatorname{sgn}(v) \partial_{r} v(\mathbf{x}+r \omega) d r \\
& \leq|v|^{p}(\mathbf{y})+p \varepsilon^{-\frac{q}{p}} \int_{0}^{|\mathbf{x}-\mathbf{y}|}|v|^{p} d r+p \varepsilon \int_{0}^{|\mathbf{x}-\mathbf{y}|}\left|\partial_{r} v\right|^{p} d r
\end{aligned}
$$

where $\varepsilon>0$ is any constant. Let

$$
V(\mathbf{x})= \begin{cases}|v|^{p}(\mathbf{x}), & \mathbf{x} \in K \\ 0, & \mathbf{x} \notin K\end{cases}
$$


and

$$
W(\mathbf{x})= \begin{cases}\left|\partial_{r} v\right|^{p}(\mathbf{x}), & \mathbf{x} \in K \\ 0, & \mathbf{x} \notin K .\end{cases}
$$

Then, the inequality (A.13) can be rewritten as

$$
|v|^{p}(\mathbf{x}) \leq|v|^{p}(\mathbf{y})+p \varepsilon^{-\frac{q}{p}} \int_{0}^{\infty} V(\mathbf{x}+r \omega) d r+p \varepsilon \int_{0}^{\infty} W(\mathbf{x}+r \omega) d r .
$$

Integrating the above inequality with respect to $\mathbf{y}$ in $S$ yields

$$
\begin{aligned}
& |S||v|^{p}(\mathbf{x}) \leq \int_{S}|v|^{p} d S \\
& +p \int_{|\mathbf{x}-\mathbf{y}| \leq \delta}\left(\varepsilon^{-\frac{q}{p}} \int_{0}^{\infty} V(\mathbf{x}+r \omega) d r+\varepsilon \int_{0}^{\infty} W(\mathbf{x}+r \omega) d r\right) d \mathbf{y} .
\end{aligned}
$$

It is not hard to see that

$$
\begin{aligned}
\int_{|\mathbf{x}-\mathbf{y}| \leq \delta} \int_{0}^{\infty} V(\mathbf{x}+r \omega) d r d \mathbf{y} & =\int_{0}^{\infty} \int_{|\omega|=1} \int_{0}^{\delta} V(\mathbf{x}+r \omega) \rho^{d-1} d \rho d \omega d r \\
& =\frac{\delta^{d}}{d} \int_{0}^{\infty} \int_{|\omega|=1} V(\mathbf{x}+r \omega) d \omega d r \\
& =\frac{\delta^{d}}{d} \int_{K}|\mathbf{x}-\mathbf{y}|^{1-d}|v|^{p}(\mathbf{y}) d \mathbf{y}
\end{aligned}
$$

Analogously, we have

$$
\int_{|\mathbf{x}-\mathbf{y}| \leq \delta} \int_{0}^{\infty} W(\mathbf{x}+r \omega) d r d \mathbf{y}=\frac{\delta^{d}}{d} \int_{K}|\mathbf{x}-\mathbf{y}|^{1-d}\left|\partial_{r} v(\mathbf{y})\right|^{p} d \mathbf{y} .
$$

Substituting (A.15) and (A.16) into (A.14) yields

$$
\begin{aligned}
& |S||v|^{p}(\mathbf{x}) \\
\leq & \int_{S}|v|^{p} d S+\frac{p \delta^{d}}{d}\left(\varepsilon^{-\frac{q}{p}} \int_{K}|\mathbf{x}-\mathbf{y}|^{1-d}|v|^{p}(\mathbf{y}) d \mathbf{y}+\varepsilon \int_{K}|\mathbf{x}-\mathbf{y}|^{1-d}|\nabla v(\mathbf{y})|^{p} d \mathbf{y}\right) .
\end{aligned}
$$

Observe that the following holds true

$$
\int_{K}|\mathbf{x}-\mathbf{y}|^{1-d} d \mathbf{x} \leq \delta S_{d-1}
$$

where $S_{d-1}$ is the "area" of the unit surface in $\mathbb{R}^{d}$. The volume of the unit ball in $\mathbb{R}^{d}$, $\omega_{d}$, is related to $S_{d-1}$ as follows

$$
\omega_{d}=\frac{S_{d-1}}{d} .
$$

Now integrating both sides with respect to $\mathrm{x}$ in $K$ gives

$$
|S| \int_{K}|v|^{p} d K \leq|K| \int_{S}|v|^{p} d S+p \omega_{d} \delta^{d+1}\left(\varepsilon^{-\frac{q}{p}} \int_{K}|v|^{p} d K+\varepsilon \int_{K}|\nabla v|^{p} d K\right),
$$

which yields the desired estimate (A.12) by setting $\varepsilon=\left(\frac{2 p \omega_{d} d^{d+1}}{|S|}\right)^{p-1}$.

Consider a case of Lemma A.2 in which the convex domain $K$ is a shape regular $d$-simplex. Denote by $h_{K}$ the diameter of $K$. The shape regularity implies that 
1. the measure of $K$ is proportional to $h_{K}^{d}$,

2. there exists an inscribed ball $B_{K} \subset K$ with diameter proportional to $h_{K}$.

Now let $S$ be a ball inside of $K$ with radius $r_{S} \geq \varsigma_{*} h_{K}$. Then, there exists a fixed constant $\kappa_{*}$ such that

$$
|K| \leq \kappa_{*}|S|
$$

Apply (A.17) in (A.12) and notice that $\tau_{*}|S| \geq h_{K}^{d}$ and $\delta=h_{K}$. Thus,

$$
\|v\|_{L^{p}(K)}^{p} \leq 2 \kappa_{*}\|v\|_{L^{p}(S)}^{p}+\left(2 p \tau_{*} h_{K} \omega_{d}\right)^{p}\|\nabla v\|_{L^{p}(K)}^{p} .
$$

For simplicity of notation, we shall rewrite (A.18) in the following form

$$
\|v\|_{K, p}^{p} \leq a_{0}\|v\|_{S, p}^{p}+a_{1} h_{K}^{p}\|\nabla v\|_{K, p}^{p} .
$$

If $v$ is infinitely smooth, then a recursive use of the estimate (A.19) yields the following result

$$
\|v\|_{K, p}^{p} \leq \sum_{j=0}^{n} a_{j} h_{K}^{j p}\left\|\nabla^{j} v\right\|_{S, p}^{p}+a_{n+1} h_{K}^{p n+p}\left\|\nabla^{n+1} v\right\|_{K, p}^{p} .
$$

In particular, if $v$ is a polynomial of degree $n$, then

$$
\|v\|_{K, p}^{p} \leq \sum_{j=0}^{n} a_{j} h_{K}^{j p}\left\|\nabla^{j} v\right\|_{S, p}^{p}
$$

The standard inverse inequality implies that

$$
\left\|\nabla^{j} v\right\|_{S, p} \lesssim h_{K}^{-j}\|v\|_{S, p} .
$$

Substituting the above into (A.21) gives

$$
\|v\|_{K, p}^{p} \lesssim\|v\|_{S, p}^{p} .
$$

The result is summarized as follows.

Lemma A.3 (Domain Inverse Inequality). Let $K \subset \mathbb{R}^{d}$ be a d-simplex which has diameter $h_{K}$ and is shape regular. Assume that $S$ is a ball in $K$ with diameter $r_{S}$ proportional to $h_{K}$; i.e., $r_{S} \geq \varsigma_{*} h_{K}$ with a fixed $\varsigma_{*}>0$. Then, there exists a constant $C=C\left(\varsigma_{*}, n\right)$ such that

$$
\|v\|_{L^{p}(K)} \leq C\left(\varsigma_{*}, n\right)\|v\|_{L^{p}(S)}
$$

for any polynomial $v$ of degree no more than $n$.

A.4. Inverse Inequalities. The usual inverse inequality in finite element analysis also holds true for piecewise polynomials defined on the finite element partition $\mathcal{T}_{h}$ provided that it satisfies the assumptions A1-A4.

Lemma A.4. Let $\mathcal{T}_{h}$ be a finite element partition of $\Omega$ consisting of polygons or polyhedra. Assume that $\mathcal{T}_{h}$ satisfies all the assumptions A1-A4 and $p \geq 1$ be any real number. Then, there exists a constant $C=C(n)$ such that

$$
\|\nabla \varphi\|_{T, p} \leq C(n) h_{T}^{-1}\|\varphi\|_{T, p}, \quad \forall T \in \mathcal{T}_{h}
$$


for any piecewise polynomial $\varphi$ of degree $n$ on $\mathcal{T}_{h}$.

Proof. The proof is merely a combination of Lemma A.3 and the standard inverse inequality on d-simplices. To this end, for any $T \in \mathcal{T}_{h}$, let $S(T)$ be the circumscribed simplex that is shape regular. It follows from the standard inverse inequality that

$$
\|\nabla \varphi\|_{T, p} \leq\|\nabla \varphi\|_{S(T), p} \leq C h_{T}^{-1}\|\varphi\|_{S(T), p} .
$$

Then we use the estimate (A.23), with $K=S(T)$, to obtain

$$
\|\nabla \varphi\|_{T, p} \leq C h_{T}^{-1}\|\varphi\|_{S, p} \leq C h_{T}^{-1}\|\varphi\|_{T, p},
$$

where $S$ is a ball inside of $T$ with a diameter proportional to $h_{T}$. This completes the proof of the lemma.

Lemma A.5. Let $\mathcal{T}_{h}$ be a finite element partition of $\Omega$ consisting of polygons or polyhedra. Assume that $\mathcal{T}_{h}$ satisfies all the assumptions A1-A4 and $p \geq r \geq 1$ be any two real numbers. Then, there exists a constant $C=C(n)$ such that

$$
\|\varphi\|_{L^{p}(\Omega)} \leq C h^{\frac{d}{p}-\frac{d}{r}}\|\varphi\|_{L^{r}(\Omega)}
$$

for any piecewise polynomial $\varphi$ of degree $n$ on $\mathcal{T}_{h}$.

Proof. For any $T \in \mathcal{T}_{h}$, let $S(T)$ be the circumscribed simplex that is shape regular. It follows from the standard inverse inequality that

$$
\|\varphi\|_{T, p} \leq\|\varphi\|_{S(T), p} \leq C h_{T}^{\frac{d}{p}-\frac{d}{r}}\|\varphi\|_{S(T), r} .
$$

We then use the estimate (A.23), with $K=S(T)$, to obtain

$$
\begin{aligned}
\|\varphi\|_{T, p} & \leq C h_{T}^{\frac{d}{p}-\frac{d}{r}}\|\varphi\|_{S, r} \\
& \leq C h_{T}^{\frac{d}{p}-\frac{d}{r}}\|\varphi\|_{T, r},
\end{aligned}
$$

where $S$ is a ball inside of $T$ with a diameter proportional to $h_{T}$. It follows from (A.26) that

$$
\begin{aligned}
\|\varphi\|_{L^{p}(\Omega)}^{p} & =\sum_{T \in \mathcal{T}_{h}}\|\varphi\|_{T, p}^{p} \\
& \leq C h^{d-\frac{p d}{r}} \sum_{T \in \mathcal{T}_{h}}\|\varphi\|_{T, r}^{p} .
\end{aligned}
$$

Since $\frac{r}{p} \leq 1$, then we have from the above inequality that

$$
\begin{aligned}
\|\varphi\|_{L^{p}(\Omega)}^{r} & \leq C h^{\frac{r d}{p}-d}\left(\sum_{T \in \mathcal{T}_{h}}\|\varphi\|_{T, r}^{p}\right)^{\frac{r}{p}} \\
& \leq C h^{\frac{r d}{p}-d} \sum_{T \in \mathcal{T}_{h}}\|\varphi\|_{T, r}^{r},
\end{aligned}
$$

which implies the desired inverse inequality (A.25). 


\section{REFERENCES}

[1] D. N. ARnold AND F. BrezzI, Mixed and nonconforming finite element methods: implementation, postprocessing and error estimates, RAIRO Modl. Math. Anal. Numr., 19(1), (1985), pp. 7-32.

[2] S. BREnNER AND L. Sung, $C^{0}$ interior penalty methods for fourth order elliptic boundary value problems on polygonal domains, J. Sci. Comput., (2005), pp. 83-118.

[3] G. Engel, K. Garikipati, T. Hughes, M.G. Larson, L. Mazzei, and R. Taylor, Continuous/discontinuous finite element approximations of fourth order elliptic problems in structural and continuum mechanics with applications to thin beams and plates, and strain gradient elasticity, Comput. Meth. Appl. Mech. Eng., 191 (2002), pp. 3669-3750.

[4] R. FALK Approximation of the biharmonic equation by a mixed finite element method, SIAM J. Numer. Anal. 15 (1978), pp. 556-567.

[5] T. Gudi, N. Nataraj, A. K. Pani, Mixed Discontinuous Galerkin Finite Element Method for the Biharmonic Equation, J Sci Comput, 37 (2008), pp. 139-161.

[6] L.S.D. MORLEY, The triangular equilibrium element in the solution of plate bending problems, Aero. Quart., 19 (1968), pp. 149-169.

[7] P. Monk, A mixed finite element methods for the biharmonic equation, SIAM J. Numer. Anal. 24 (1987), pp. 737-749.

[8] I. Mozolevski ANd E. Sli, Bsing, P.R.: hp-Version a priori error analysis of interior penalty discontinuous Galerkin finite element approximations to the biharmonic equation, J. Sci. Comput. 30 (2007), pp. 465-491.

[9] L. Mu, J. WANG, AND X. YE, Weak Galerkin finite element methods on polytopal meshes, arXiv: $1204.3655 \mathrm{v} 2$.

[10] L. Mu, Y. Wang, J. WAng And X. Ye, A weak Galerkin mixed finite element method for biharmonic equations. arXiv:1210.3818v2.

[11] J. WANG AND X. YE, A weak Galerkin finite element method for second-order elliptic problems, J. Comp. and Appl. Math, 241 (2013), pp. 103-115. arXiv:1104.2897v1.

[12] J. WANG AND X. YE, A weak Galerkin mixed finite element method for second-order elliptic problems, arXiv:1202.3655v2.

[13] J. WAng AND X. YE, A weak Galerkin finite element method for the Stokes equations, arXiv:1302.2707v1. 